\title{
Characterization of the cryptic Escherichia lineages: rapid identification and prevalence
}

\author{
Olivier Clermont, ${ }^{1,2}$ David M. Gordon, ${ }^{3}$ \\ Sylvain Brisse, ${ }^{4}$ Seth T. Walk ${ }^{5}$ and Erick Denamur ${ }^{1,2 *}$ \\ ${ }^{1}$ INSERM, UMR-S 722, Paris, F-75018, France. \\ ${ }^{2}$ Univ Paris Diderot, Sorbonne Paris Cité, UMR-S 722, \\ Faculté de Médecine, Site Xavier Bichat, Paris, \\ F-75018, France. \\ ${ }^{3}$ Research School of Biology, Australian National \\ University, Canberra, ACT 0200, Australia. \\ ${ }^{4}$ Genotyping of Pathogens and Public Health, Institut \\ Pasteur, 75724 Paris, France. \\ ${ }^{5}$ Department of Internal Medicine, University of Michigan \\ Health System, 4618 Medical Science Building II, Ann \\ Arbor, MI 48109, USA.
}

\section{Summary}

Strains phenotypically indistinguishable from Escherichia coli and belonging to at least five distinct cryptic lineages, named Escherichia clades I to V, that are genetically divergent from $E$. coli yet members of the genus have been recently found using multi-locus sequence typing (MLST). Very few epidemiological data are available on these strains as their detection by MLST is not suitable for large-scale studies. In this work, we developed a rapid PCR method based on aes and chuA allele-specific amplifications that assigns a strain a cryptic lineage membership. By screening more than $\mathbf{3 5 0 0}$ strains with this approach, we show that the cryptic lineages of Escherichia are unlikely to be detected in human faecal samples (2-3\% frequency) and even less likely to be isolated from extra-intestinal body sites $(<1 \%$ frequency). They are more abundant in animal faeces ranging from $3-8 \%$ in non-human mammals to $8-28 \%$ in birds. Overall, the strains from the clade $\mathrm{V}$ are the most abundant and from the clade II very rare. These results suggest that members of the cryptic clades are unlikely to be of significance to human and health but may influence the use of ' $E$. coli' as an indicator of water quality.

Received 2 March, 2011; accepted 2 May, 2011. *For correspondence. E-mail erick.denamur@inserm.fr; Tel. (+33) 1572775 34; Fax (+33) 157277521 .

\section{Introduction}

Escherichia coli was, until recently, considered to be the best-known bacterial species. First, because the E. coli strain $\mathrm{K}-12$ has been used widely as a model organism in genetic and molecular biology studies (Neidhardt et al., 1996). Second, because population geneticists have used $E$. coli to test a variety of hypotheses with each new technological advance (reviewed in Tenaillon et al., 2010). Third, because $E$. coli is a very significant human and animal pathogen it has been the subject of intense scrutiny by the medical community. In nature, E. coli encompasses strains living as commensals inhabiting the vertebrate gut (primary habitat) but also intra-and extraintestinal pathogenic strains (Kaper et al., 2004; Tenaillon et al., 2010). Soil, water and sediments represent the species' secondary habitat, and E. coli cells may be as abundant in the secondary habitat as in the primary habitat (Savageau, 1983). The genetic structure of the species is predominantly clonal, meaning that recombination is rare enough to allow the long-term propagation of clonal lineages (Tenaillon et al., 2010). Phylogenetic analyses initially revealed at least five main phylogenetic groups (A, B1, B2, D and E) (Gordon et al., 2008). Recently, an additional group of strains close to B2 phylogroup strains and called $F$ has been delineated (Jaureguy et al., 2008). At the genomic level, E. coli is highly diverse with 10 times more genes in the pan-genome (the total number of genes in the species) than in the core genome (the genes present in all the strains) (Rasko et al., 2008; Touchon et al., 2009). Of the other species of Escherichia, $E$. fergusonii is the species genetically most similar to $E$. coli (Lawrence et al., 1991; Walk et al., 2009) and, while less similar, E. albertii is also clearly a member of the genus (Hyma et al., 2005; Walk et al., 2009). Escherichia blattae, E. hermanii and E. vulneris share minimal genetic similarity with other Escherichia and they should not be considered valid members of the genus (Lawrence et al., 1991; Hartl, 1992; Cilia et al., 1996; Paradis et al., 2005; Pham et al., 2007; Priest and Barker, 2010).

Given that $E$. coli has been the subject of intense study for over a century, it was perhaps surprising when strains phenotypically indistinguishable from $E$. coli were found to be genetically divergent from E. coli (Walk et al., 2009). Multi-locus sequence analysis based on 22 core genome 
genes revealed that these strains fell into five novel 'cryptic' lineages that were clearly members of the genus, but which are distinct from the three named Escherichia species. Clade I is very closely related to $E$. coli, whereas clade V strains are the most divergent. Clades III and IV are sister groups, branched between E. coli and clade V. Only one strain of clade II has been reported (Walk et al., 2009). It has been suggested that the primary niche of these strains could be habitats outside of the host gastrointestinal tract (Walk et al., 2009; Ingle et al., 2011), which might explain why they have not been found before, as much of our understanding of $E$. coli is based on faecal and clinical isolates.

We know little of the distribution or the ecological characteristics of these novel Escherichia lineages. This lack of understanding is largely due to the fact that, at present, the only method by which members of the cryptic clades can be distinguished from $E$. coli is by obtaining nucleotide sequence data for an informative gene. Consequently, we developed a PCR-based method that distinguishes between strains of $E$. coli and members of the novel clades and which allows most cryptic strains to be assigned to one of the novel clades. Using this approach, we then screened various collections of commensal and pathogenic strains in order to determine their ecological distribution.

\section{Results}

\section{Multi-locus sequence typing (MLST) and single} gene phylogenies

Characterization of 419 strains isolated from humans and other animals living in France and from animals living in various parts of the world, using the Institute Pasteur MLST scheme (Le Gall et al., 2007; Jaureguy et al., 2008), revealed 37 Escherichia strains that could not be considered typical E. coli. In order to determine the clade membership of these non-E. coli strains as in (Walk et al., 2009), a representative subset of strains was characterized using the Achtman MLST scheme (Wirth et al., 2006). Trees based on the concatenated data of each of the MLST schemes, including typical E. coli, E. fergusonii and $E$. albertii strains and rooted on Salmonella, were largely congruent (Fig. 1). Both schemes identified clades $\mathrm{I}-\mathrm{V}$ as distinct from $E$. coli, with clade I between $E$. coli and E. fergusonii. However, the phylogeny inferred using the Achtman MLST scheme indicates that the strain ROAR19 is a clade II strain, while the Institute Pasteur scheme depicts ROAR19 as more closely related to $E$. coli than the clade II strain B1147 (Fig. 1). Further, using the Institute Pasteur scheme, E. albertii strains appear as the most basal branch whereas it is the clade $\mathrm{V}$ strains that are basal using the Achtman MLST scheme. It can be noted that all the nodes of the Institute Pasteur scheme tree are supported by high bootstrap values, which is not the case with the other scheme (Fig. 1). This could be due in part to the fact that the Institute Pasteur scheme is based on a greater number of nucleotides than the Achtman MLST scheme (7032 versus 3423).

Walk and colleagues (2009) reported that only three genes, Iys $P$, rpoS and fum $C$, appeared capable of unambiguously assigning all Escherichia strains to the appropriate cryptic clade or species of Escherichia. Recently, it has been shown that aes, the gene encoding esterase $B$, is a powerful phylogenetic marker of $E$. coli (Lescat et al., 2009). We sequenced the aes gene in a set of 29 representative non-E. coli strains as well as E. fergusonii and $E$. albertii strains. The phylogenetic tree inferred using these sequences, as well as with sequences from $E$. coli strains belonging to the five main phylogenetic groups, shows that this gene is able to clearly delineate the five cryptic clades from E. coli, E. albertii and E. fergusonii, with two exceptions. There are two identical clade I strains (H442 and TW10509), identified as such based on the MLST data (Fig. 1), having aes sequences that are more like E. fergusonii aes sequences rather than the aes sequences of other clade I strains (Fig. 2). The strain $\mathrm{B} 2 \mathrm{H} 5$ is assigned to $E$. coli based on the MLST data; however, its aes gene is clearly more like the aes genes of clade I strains than those of E. coli (Fig. 2).

It can be concluded from these analyses that various lineages of Escherichia are phylogenetically robust and can be identified by classical MLST schemes. It has also been shown that, provided the appropriate gene is chosen, nucleotide sequence data for a single gene can reliably assign an Escherichia strain to the appropriate lineage. However, a sequencing approach for epidemiological screening is tedious and relatively expensive. Consequently, the goal was to develop a simple PCR-based approach to both identify strains belonging to the cryptic clades and assign these strains to the appropriate clade.

\section{Preliminary identification of cryptic Escherichia lineages}

The MLST results identify a group of strains that are unambiguously members of one of the five cryptic clades of Escherichia. The characteristics of these strains were used to discover which traits might indicate that a strain in a collection of isolates phenotypically resembling $E$. coli could be a member of one the cryptic clades.

It is now common practice to use the triplex PCR method (Clermont et al., 2000) to assign E. coli strains to the phylo-groups $\mathrm{A}, \mathrm{B} 1, \mathrm{~B} 2$ or $\mathrm{D}$. This method is based on the amplification of three DNA fragments, named chuA, $y j a A$ and TSPE4.C2, belonging to $c h u A$, yjaA and a putative lipase esterase genes respectively (Clermont et al., 2000; Gordon et al., 2008). The different combinations of 

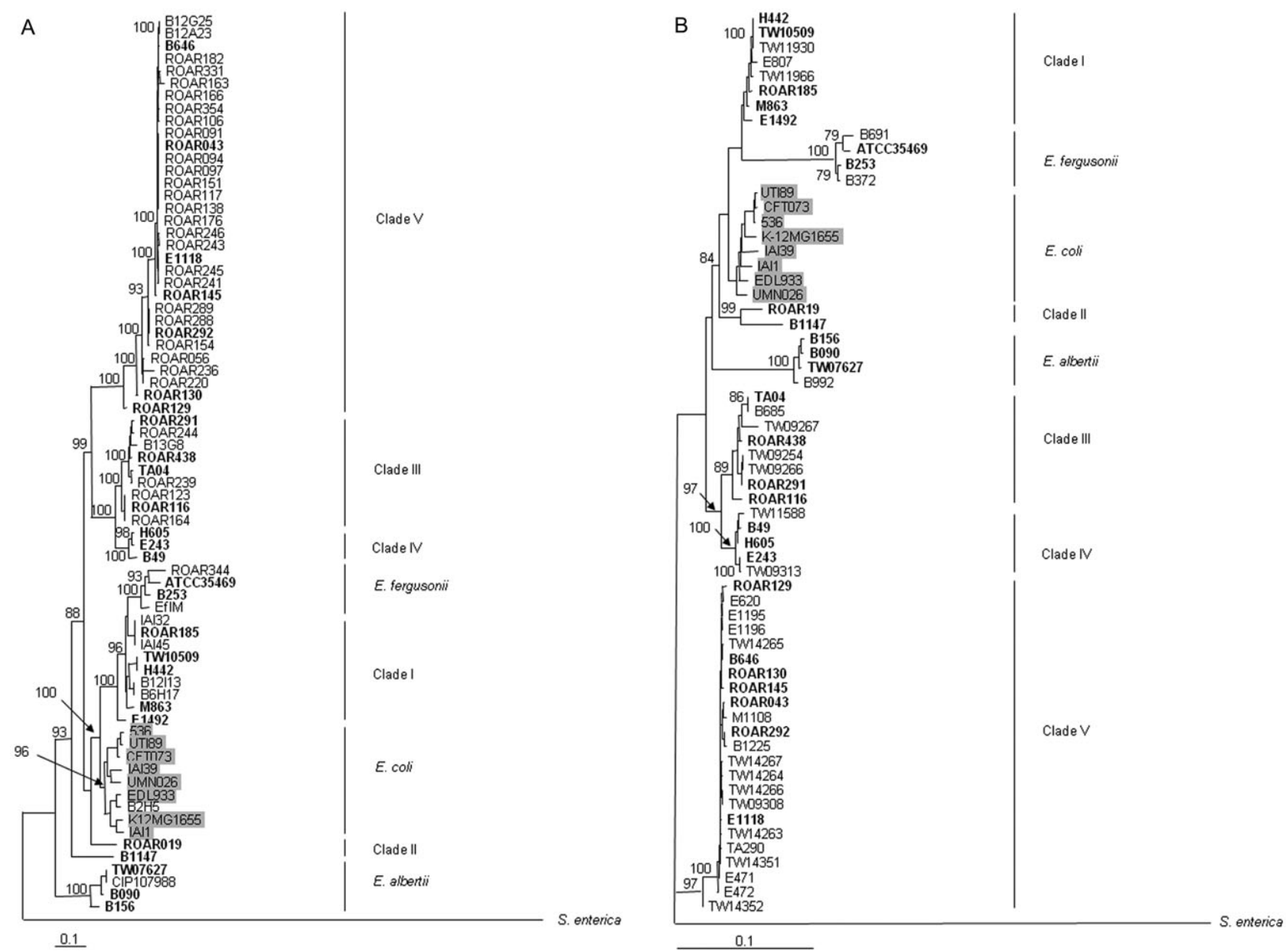

Fig. 1. Phylogenetic trees of Australian and French representative commensal and pathogenic Escherichia clade strains as well as representative strains of E. coli, E. fergusonii and E. albertii reconstructed from (A) 7 complete genes (7032 bp) (Le Gall et al., 2007) and (B) 7 partial genes (3423 bp) (Wirth et al., 2006) using PHYML (Guindon et al., 2005). The trees are rooted on Salmonella strains. Bootstrapping was performed on 500 replicates and values above 70 for the major nodes are indicated. Strains common to the aes and chuA trees (Figs 2 and 3 respectively) are in bold. E. coli strains are boxed in grey. The strain B2H5, which has an ambiguous status, is not boxed.

these DNA fragments give rise to 7 genotypes: $A_{0}$ and $A_{1}$ belonging to the $\mathrm{A}$ phylo-group, $\mathrm{B} 1, \mathrm{~B}_{2}$ and $\mathrm{B} 2_{3}$ belonging to the $B 2$ phylo-group and $D_{1}$ and $D_{2}$ belonging to the $D$ phylo-group (Gordon et al., 2008). The majority (88\%) of strains belonging to clades II, III, IV or V failed to yield any of the expected triplex method PCR products $\left(A_{0}\right.$ genotype) (Table 1). Although most clade I strains are positive for chuA and $y j a A$ ( $B 2_{2}$ genotype), clade I strains can have a variety of genotypes (Table 1). Although cryptic clade strains could not be distinguished from $E$. coli strains using biochemical and enzymatic reaction patterns analysed by multidimensional scaling (Walk et al., 2009; Sabarly et al., 2011), we observed specific patterns for lysine and ornithine utilization. The majority of $E$. coli are positive for lysine and ornithine catabolism (Ewing, 1986). Most clade III and IV strains are lysine negative and ornithine positive, while all but one clade $\mathrm{V}$ strains are lysine positive and ornithine negative (Table 1). The two clade II strains are also lysine positive and ornithine negative. These traits fail to distinguish clade I strains from E. coli.

Some cryptic clade strains yielded a chuA product using the triplex PCR method and genome analysis revealed that chuA was present in E. albertii. These results suggested that chuA may be present in all of the cryptic lineages, but divergent from chuA of $E$. coli, and consequently the chuA PCR was performed at $50^{\circ} \mathrm{C}$ of annealing [instead of $55^{\circ} \mathrm{C}$ in the classical Clermont method (Clermont et al., 2000)]. PCR screening using this approach revealed that although some clade I strains (38\%) lack chuA, all clade III, IV and V strains were chuA positive, while the clade II strains are chuA negative. A phylogenetic tree inferred using the complete chuA sequences of 27 strains from clades I, III, IV and V as well as $E$. coli and rooted on the $E$. albertii sequences indicated that the chuA phylogeny is congruent with the phy- 


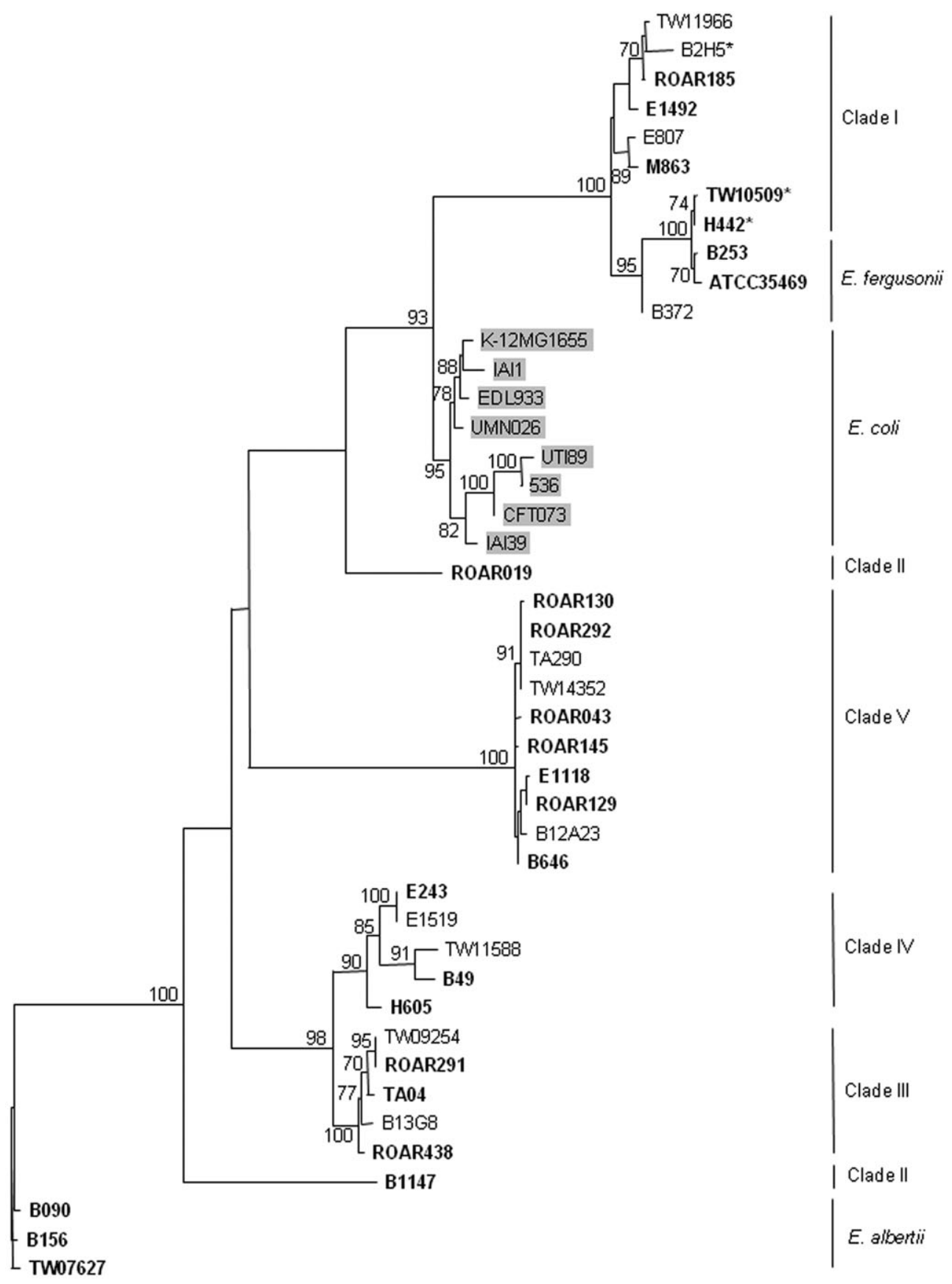

Fig. 2. Phylogenetic tree of Escherichia clade, E. coli and E. fergusonii strains reconstructed from the 894 bp of the aes gene using PHYML (Guindon et al., 2005). The tree is rooted on the E. albertii strains. Bootstrapping was performed on 500 replicates and values above 70 are indicated at the nodes. Strains in bold correspond to the strains present also in Fig. 1 and/or 3. E. coli strains are boxed in grey. The strains noted with a star (B2H5, TW10509, H442) correspond to strains having a different position in the MLST trees (Fig. 1).

logenetic history of the strains (Fig. 3), as previously observed for E. coli (Gordon et al., 2008). The clade I strains except E1492 are monophyletic, however they fall within $E$. coli between the $\mathrm{B} 2 / \mathrm{F}$ and $\mathrm{D} / \mathrm{E}$ phylo-group strains.
A rapid $P C R$ method to assign a strain a cryptic clade membership

The complete aes and chuA nucleotide sequence data were used to develop an allele-specific PCR of the aes 
Table 1. PCR triplex genotypes and metabolic properties of strains belonging to the cryptic lineages of Escherichia isolated from Australia, France and other parts of the world.

\begin{tabular}{|c|c|c|c|c|c|}
\hline Trait & Clade I $(n=13)$ & Clade II $(n=2)$ & Clade III $(n=14)$ & Clade IV $(n=6)$ & Clade $\vee(n=52)$ \\
\hline \multicolumn{6}{|c|}{$\begin{array}{l}\text { Triplex PCR }{ }^{\mathrm{a}} \\
\text { chuA yjaA TSPE4.C2 genotype }\end{array}$} \\
\hline$---A_{0}$ & 0 & 1 & 12 & 4 & 48 \\
\hline$-+-A_{1}$ & 5 & 1 & 0 & 0 & 0 \\
\hline$--+B 1$ & 0 & 0 & 1 & 1 & 3 \\
\hline$+++\mathrm{B} 2_{3}$ & 1 & 0 & 0 & 0 & 1 \\
\hline$++-B 2_{2}$ & 7 & 0 & 0 & 0 & 0 \\
\hline$+--D_{1}$ & 0 & 0 & 1 & 1 & 0 \\
\hline$+-+D_{2}$ & 0 & 0 & 0 & 0 & 0 \\
\hline \multicolumn{6}{|c|}{$\begin{array}{l}\text { Catabolism } \\
\text { Iysine, ornithine }\end{array}$} \\
\hline++ & 10 & 0 & 0 & 1 & 1 \\
\hline+- & 1 & 2 & 0 & 0 & 51 \\
\hline-+ & 2 & 0 & 11 & 4 & 0 \\
\hline-- & 0 & 0 & 3 & 1 & 0 \\
\hline
\end{tabular}

a. As in Clermont and colleagues (2000).

+ , presence of the PCR product; -, absence of the PCR product.

and chuA genes based on clade-specific single nucleotide polymorphisms that allows the amplification of PCR products of different sizes according to the Escherichia clade (clades I and II and clades III, IV and V respectively). The primers and the lengths of the PCR products are given Table 2 and an example of the PCR products migrated in an agarose gel is shown in Fig. 4. The robustness of this method, first developed in Erick Denamur's lab (France) was also tested in the David Gordon's lab (Australia) on a panel of strains previously characterized with the MLST data. In total, 14 clade III, 6 clade IV and 52 clade V strains yield chuA PCR products of the expected size, while 13 clade I and 2 clade II strains yielded aes products of the expected size. No positive signal was obtained using these allele-specific primers neither in a collection of $11 \mathrm{E}$. albertii strains nor in the ECOR collection strains (Ochman and Selander, 1984) that are representative of the E. coli genetic diversity. However, the three E. fergusonii strains tested yielded a product using the aes clade I primers. This outcome was expected given the close similarity of aes for clade I and E. fergusonii strains (Fig. 2).

According to the classical triplex PCR results of the clade strains (Table 1), we propose a two-step screening strategy based first on the triplex PCR amplifying chuA, yja $A$ and TSPE4.C2 (Clermont et al., 2000) followed by the allele-specific PCR on $\mathrm{A}_{0}$ (chuA, yjaA and TSPE4.C2 negative) and $\mathrm{B}_{2}$ (chuA and $y j a A$ positive and TSPE.4C2 negative) genotypes.

\section{Relative abundance of strains belonging to the cryptic clades}

The MLST analysis of bacterial isolates identified as ' $E$. coli' based on classic phenotypic characteristics should provide an unbiased estimate of the fraction of strains that are not $E$. coli, but rather members of one of the cryptic clades. The MLST data indicate that the relative abundance of members of the cryptic clades in an ' $E$. coli' collection will depend on the source of the isolate (Table 3). The frequency of strains belonging to the cryptic clades ranged from $0 \%$ among extra-intestinal isolates to $28 \%$ for isolates from birds. There also appears to be a locality effect, as the frequency of the cryptic clade strains in the collection of French isolates was $8.8 \%$ but only $4.4 \%$ in the collection of Australian isolates (contingency analysis, likelihood ratio $\chi^{2}=6.74, P>\chi^{2}=0.009$ ).

The rarity of strains belonging to the cryptic lineages among extra-intestinal isolates from humans was confirmed when strains from two collections of largely clinical isolates were screened using the allele-specific PCR approach: 65 strains isolated in the 1980s from patients living in France (Picard et al., 1999) and 1081 strains isolated from septicaemic patients in 2005 during the COLIBAFI study in France (Lefort et al., 2011). The 1980's collection yielded five strains with an A profile and $4 \mathrm{~B}_{2}$ strains and two of these strains were members of clade I. The COLIBAFI collection contained $98 \mathrm{~A}_{0}$ and 56 $\mathrm{B} 2_{2}$ strains, two of these were clade I strains, two were clade $\mathrm{V}$ and one a member of clade III. Thus, the frequency of strains belonging to the cryptic lineages in these two collections was $3.1 \%$ and $0.5 \%$ respectively.

One of the $\mathrm{B}_{2}$ strain (B2H5) from the COLIBAFI collection, screened using the clade specific chuA primers, yielded a PCR product with the size expected for a clade I strain. The nucleotide sequence of the chuA gene for $\mathrm{B} 2 \mathrm{H} 5$ confirmed that this strain clustered with other clade I strains (Fig. 3). The nucleotide sequence of the aes gene for $\mathrm{B} 2 \mathrm{H} 5$ also would suggest that it is a clade I strain (Fig. 2). However, the MLST results for this strain has it 


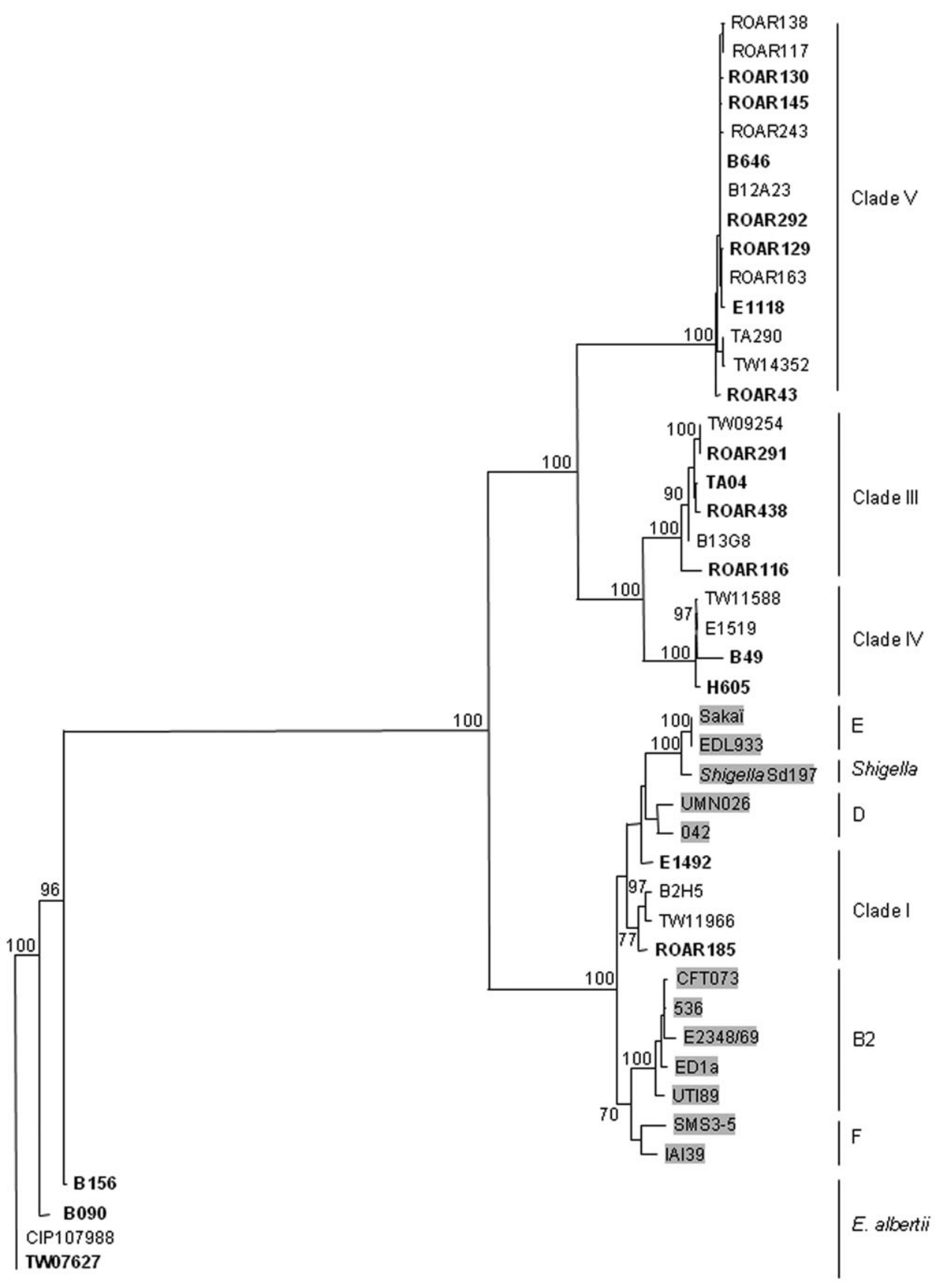

0.1

Fig. 3. Phylogenetic tree of Escherichia clade, E. coli and E. fergusonii strains reconstructed from the $1983 \mathrm{bp}$ of the complete chuA gene using PHYML (Guindon et al., 2005). The tree is rooted on the E. albertii strains. Bootstrapping was performed on 500 replicates and values above 70 are indicated at the nodes. Strains in bold correspond to the strains present also in Fig. 1 and/or 2. E. coli/Shigella strains are boxed in grey. The strain $\mathrm{B} 2 \mathrm{H} 5$, which has an ambiguous status, is not boxed.

clearly clustering with $E$. coli and it is most similar to a phylo-group E strain (Fig. 1A).

Further screening of Australian isolates identified as $E$. coli using the clade specific PCR revealed additional members of the cryptic Escherichia lineages. These PCR screening data combined with the MLST results allow the relative abundance of strains of the various cryptic lineages to be assessed in France and Australia. Strains belonging to clade II are rare, while clade $\mathrm{V}$ strains are the most common of the cryptic clades (Table 4). Strains belonging to the other clades are of intermediate abundance and their relative frequency may vary with locality, 
Table 2. chuA and aes primers used in the study for the allele-specific PCR amplifications.

\begin{tabular}{|c|c|c|c|}
\hline Primer designation & Primer sequence & Target & Size of PCR product (bp) \\
\hline aesl.1 & 5'-ССТСТАСТСАСССАAАAGTC-3' & aes & 315 \\
\hline aesl.2 & 5'-ATCACGTAACCACAACGCAC-3' & & \\
\hline aesll.1 & 5'-CGCCTGTTGTCACTTCCACG-3' & aes & 125 \\
\hline aesIl.2 & 5'-GTTTATCACGCAGCCACAAG-3' & & \\
\hline chulll.1 & 5'-GTGTTGAGATTGTCCGTGGG-3' & chuA & 183 \\
\hline chulll.2 & 5'-CAAAAGCACTGGCGCCCAG-3' & & \\
\hline chulV.1 & 5'-CTGGCGAAAGGAACCTGGA-3' & chuA & 461 \\
\hline chulV.2 & 5'-GTTATCTCATCTTGCAGCCAA-3' & & \\
\hline chuV.1 & 5'-ACTGTATGGCAGTGGCGCAT-3' & chuA & 600 \\
\hline chuV.2 & 5'-GCAAAACTATCGGCAAACAGC-3' & & \\
\hline
\end{tabular}

as it appears that clade IV strains are less common in France than clade III strains while the opposite is true for isolates from Australia (contingency analysis, likelihood ratio $\left.\chi^{2}=11.93, P<\chi^{2}=0.018\right)$ (Table 4$)$.

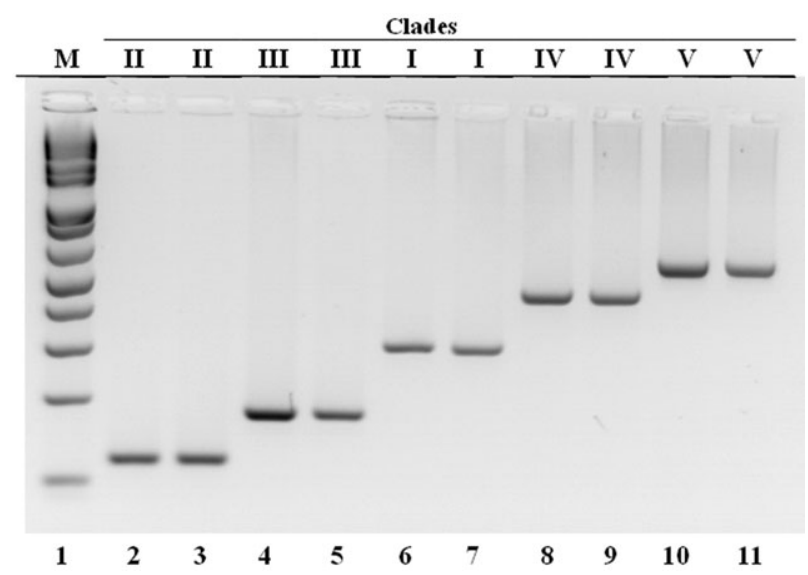

Fig. 4. Allele-specific PCR amplifications of the Escherichia clade I-V strains. The PCR products were loaded on a $2 \%$ agarose gel. Lane 1 (MW): molecular weight marker (1 kb Plus DNA Ladder, Invitrogen); lanes 2 and 3 (Clade II strains): ROAR19 and B1147 respectively; lanes 4 and 5 (Clade III strains): ROAR116 and ROAR438 respectively; lanes 6 and 7 (Clade I strains): IAI32 and ROAR185 respectively; lanes 8 and 9 (Clade IV strains): B49 and E243 respectively; lanes 10 and 11 (Clade V strains): ROAR43 and ROAR129 respectively.

\section{Discussion}

Although E. fergusonii and E. albertii strains are quite easily distinguished from E. coli (Farmer et al., 1985; Oaks et al., 2010), there is no known suite of phenotypic traits capable of distinguishing among all Escherichia lineages. Consequently, the recently discovered cryptic lineages of $E$. coli can only be discriminated from each other and from $E$. coli using genetic data. Although, nucleotide sequence data for a single gene, such as fumC and aes, can determine the lineage membership of an isolate, such an approach is not suitable for large-scale studies. The PCR-based screening method offers a simple method of determining if an isolate with the phenotypic characteristics of $E$. coli is actually a member of one of the cryptic Escherichia clades and to what clade it belongs. The method appears to be very accurate for strains belonging to clades II-V.

The method for clade I identification may yield false positives as suggested by the results obtained for the strain B2H5. The MLST data (7032 bp) clearly indicate $\mathrm{B} 2 \mathrm{H} 5$ is an $E$. coli strain (Fig. 1), while the nucleotide sequence data for chuA and aes (2877 bp for both genes) would suggest that it is a clade I strain (Figs 2 and 3 ). Walk and colleagues (2009) found evidence of allele sharing between clade I strains and E. fergusonii, as we observed for the H442 and TW10509 aes alleles (Fig. 2),

Table 3. The frequency of strains belonging to the cryptic lineages of Escherichia as determined by multi-locus sequence analysis.

\begin{tabular}{|c|c|c|c|c|}
\hline \multirow[b]{2}{*}{ Source } & \multicolumn{2}{|r|}{ France } & \multicolumn{2}{|r|}{ Australia } \\
\hline & Number examined & Number of clade members (\%) & Number examined & Number of clade members (\%) \\
\hline Soil, water, sediment & 0 & ND & 99 & $8(8.1)$ \\
\hline Fish/reptiles & 0 & ND & 29 & $0(0)$ \\
\hline Mammals & 279 & $23(8.2)$ & 124 & $4(3.2)$ \\
\hline Birds & 39 & $11(28.2)$ & 77 & $6(7.8)$ \\
\hline Human (faecal) & 101 & $2(2.0)$ & 60 & $2(3.3)$ \\
\hline Human $\left(\right.$ clinical) ${ }^{a}$ & 0 & ND & 58 & $0(0)$ \\
\hline Source effect ${ }^{b}$ & $P>\chi^{2}<0.001$ & & $P>\chi^{2}=0.024$ & \\
\hline
\end{tabular}

a. Extra-intestinal isolates.

b. Contingency analysis, likelihood ratio $\chi^{2}$. 
Table 4. Relative abundance of the five cryptic lineages of Escherichia.

\begin{tabular}{lcr}
\hline Clade & $\begin{array}{l}\text { France } \\
n(\%)\end{array}$ & \multicolumn{1}{c}{$\begin{array}{l}\text { Australia } \\
n(\%)\end{array}$} \\
\hline Clade I & $6(13.6)$ & $5(19.2)$ \\
Clade II & 0 & $1(3.8)$ \\
Clade III & $8(18.2)$ & $2(7.7)$ \\
Clade IV & 0 & $4(15.4)$ \\
Clade V & $30(68.2)$ & $14(53.8)$ \\
\hline
\end{tabular}

and a recent paper by Luo and colleagues (2011) demonstrates that more recombination events are detected occurring between strains belonging to clade I and E. coli than between clade I strains and strains belonging to the other Escherichia clades.

Although, it would be ideal if all strains with a phenotype resembling that of $E$. coli were screened using the new PCR method, a more cost-effective approach would be to restrict the screening to those strains yielding the triplex PCR method genotypes of $A_{0}$ and $B 2_{2}$. In the absence of any triplex PCR method data, then all strains that are not both lysine and ornithine positive should be screened. The problems associated with correctly identifying clade I strains and the small number of clade II representatives would suggest that all strains identified as clades I and II using the clade specific PCR method should have their identification confirmed using one of the MLST typing schemes.

The available data indicate that strains belonging to any of the cryptic lineages of Escherichia are unlikely to be detected in human faecal samples (2-3\% frequency) and even less likely to be isolated from extra-intestinal body sites ( $<1 \%$ frequency), in agreement with the lack of intrinsic extra-intestinal virulence exhibited by these strains (Ingle et al., 2011). However, the samples reported here are from humans living in France and Australia. In humans, the relative abundance of strains belonging to the four main $E$. coli phylo-groups varies substantially with locality (Tenaillon et al., 2010). Further we have shown that when the Escherichia isolates are from birds and mammals they are more likely to be members of the cryptic clades if the hosts were sampled in France as compared with Australia. Consequently, members of the cryptic clades may be more prevalent in humans sampled in other countries.

Escherichia coli is the most common member of the Enterobacteriaceae to be isolated from mammals (Gordon and FitzGibbon, 1999); however, members of the cryptic clades can approach frequencies of $10 \%$ for isolates from non-human mammals. Escherichia coli is not prevalent in most species of birds (Gordon and Cowling, 2003), but members of the cryptic clades appear to be relatively common in birds, in both France and Australia.
Walk and colleagues (2009) argued that members of the cryptic clades might be more prevalent in water samples than in the mammalian host population, and the results of this study provide some support for this suggestion. In Australia, the cryptic clade strains are more prevalent in water samples than in faecal samples from mammals (Table 3).

These results suggest that members of the cryptic clades are unlikely to be of significance to human and health. However, the cryptic clades appear to inhabit ecological niches that may be distinct from that of most $E$. coli strains. Consequently, the cryptic clades are of significance to studies of population genetics and evolution that concern E. coli and related species. The extent to which the existence of these cryptic lineages may influence the use of ' $E$. coli' as an indicator of water quality is unknown. Further studies concerning the distribution, ecological and virulence characteristics of members of the cryptic Escherichia clades are required. It is hoped that the rapid screening method described here will lead to a better understanding of the significance of these cryptic Escherichia lineages.

\section{Experimental procedures}

\section{Bacterial strains}

MLST data were available for 419 strains characterized using the MLST scheme described at http://www.pasteur.fr/mlst/ (Jaureguy et al., 2008) modified as in Le Gall and colleagues (2007) and for 447 isolates characterized using the MLST scheme described at http://mlst.ucc.ie. (Wirth et al., 2006). Strains in these collections were identified as ' $E$. coli' using classical biochemical tests. The collection of 419 strains consisted of 101 isolates taken from the faeces of humans living in France as well as 279 isolates from mammals and 39 isolates from birds living in various parts of the world (Skurnik et al., 2006). The collection of 447 strains were obtained from hosts living in Australia and consisted of 60 faecal and 58 extra-intestinal isolates from humans, together with 124 faecal isolates from mammals, 77 faecal isolates from birds, 29 faecal isolates from reptiles, and 99 isolates from soil sediment or water samples (Gordon et al., 2008). The MLST analysis allowed members of the cryptic Escherichia clades to be identified (Walk et al., 2009) and these strains served as the positive controls for the development of the rapid PCRbased screening methods whereas the others served as negative controls. The strains described by Walk and colleagues (2009) were also included in this study.

To gain a better understanding of the distribution and abundance of strains belonging to the cryptic clades of Escherichia, several previously published collections of strains identified as $E$. coli by classical biochemical tests were screened using the rapid PCR screening protocols. (i) A collection of 15 faecal and 67 extra-intestinal isolates collected in France during the 1980s (Picard et al., 1999). (ii) A collection of 1081 strains isolated from septicaemic patients in 2005 during the COLIBAFI study in France (Lefort et al., 
2011). (iii) The Australian strains used in the MLST analyses were drawn from a much larger collection of strains obtained from hosts living in Australia that consisted of 266 faecal isolates and 353 extra-intestinal isolates from humans (Gordon et al., 2005), 12 faecal isolates from fish, 37 faecal isolates from reptiles, 134 faecal isolates from birds and 497 faecal isolates from mammals (Gordon and Cowling, 2003), together with 239 isolates from soil, sediment and water samples (Power et al., 2005). The phylo-group membership of all strains in these collections had been determined using the PCR triplex method (Clermont et al., 2000).

In addition, the strains of the ECOR collection (Ochman and Selander, 1984) and 6 E. fergusonii, 12 E. albertii and 2 Salmonella strains were used.

\section{chuA and aes gene phylogenies}

The complete chuA gene was PCR amplified and sequenced as in Gordon and colleagues (2008). The aes gene was PCR amplified and sequenced as described by Lescat and colleagues (2009). Phylogenetic analysis was performed with the maximum likelihood method, as implemented in the PHYML program (Guindon et al., 2005), with E. alberti as an outgroup.

\section{Allele-specific chuA and aes PCR detection of the Escherichia clades}

PCR reaction was carried out in a $20 \mu$ l volume containing $2 \mu$ of $10 \times$ buffer (supplied with Taq polymerase), 20 pmol of each primer, $2 \mu \mathrm{M}$ each dNTP, $1 \mathrm{U}$ of Taq polymerase (New England Biolabs, Ozyme, St Quentin-en-Yvelines, France), and $3 \mu \mathrm{l}$ of bacterial lysate or $2 \mu \mathrm{l}$ of DNA. PCR was performed with an Eppendorf Mastercycler with MicroAm tubes in the following conditions: denaturation $4 \mathrm{~min}$ at $94^{\circ} \mathrm{C}, 30$ cycles of $5 \mathrm{~s}$ at $94^{\circ} \mathrm{C}$ and $30 \mathrm{~s}$ at $63^{\circ} \mathrm{C}$, and a final extension step of $5 \mathrm{~min}$ at $72^{\circ} \mathrm{C}$. All the primers were used in the same reaction (Table 2). PCR products were loaded on $2 \%$ agarose gel with SYBR Safe DNA gel stain (Invitrogen, Cergy Pontoise, France). After electrophoresis, gels were photographed under UV light.

\section{Acknowledgements}

We are grateful to Bertrand Picard, David Skurnik and Marie Picque for their participation to the gathering and first-line characterization of some of the French collections. This work has been partially funded by the Alliance for the Prudent Use of Antibiotics (APUA) in the frame of the Reservoirs of Antibiotic Resistance (ROAR) projects 2006-2007 obtained by ED and DMG.

\section{References}

Cilia, V., Lafay, B., and Christen, R. (1996) Sequence heterogeneities among $16 \mathrm{~S}$ ribosomal RNA sequences, and their effect on phylogenetic analyses at the species level. Mol Biol Evol 13: 451-461.
Clermont, O., Bonacorsi, S., and Bingen, E. (2000) Rapid and simple determination of the Escherichia coli phylogenetic group. Appl Environ Microbiol 66: 4555-4558.

Ewing, W.H. (1986) Edwards and Ewing's Identification of Enterobacteriaceae. New York, NY, USA: Elsevier Science Publishing Co.

Farmer, J.J., 3rd, Fanning, G.R., Davis, B.R., O'Hara, C.M., Riddle, C., Hickman-Brenner, F.W., et al. (1985) Escherichia fergusonii and Enterobacter taylorae, two new species of Enterobacteriaceae isolated from clinical specimens. J Clin Microbiol 21: 77-81.

Gordon, D.M., and Cowling, A. (2003) The distribution and genetic structure of Escherichia coli in Australian vertebrates: host and geographic effects. Microbiology 149: 3575-3586.

Gordon, D.M., and FitzGibbon, F. (1999) The distribution of enteric bacteria from Australian mammals: host and geographical effects. Microbiology 145 (Pt 10): 26632671.

Gordon, D.M., Stern, S.E., and Collignon, P.J. (2005) Influence of the age and sex of human hosts on the distribution of Escherichia coli ECOR groups and virulence traits. Microbiology 151: 15-23.

Gordon, D.M., Clermont, O., Tolley, H., and Denamur, E. (2008) Assigning Escherichia coli strains to phylogenetic groups: multi-locus sequence typing versus the PCR triplex method. Environ Microbiol 10: 2484-2496.

Guindon, S., Lethiec, F., Duroux, P., and Gascuel, O. (2005) PHYML Online - a web server for fast maximum likelihoodbased phylogenetic inference. Nucleic Acids Res 33: W557-W559.

Hartl, D.L. (1992) Population genetics of microbial organisms. Curr Opin Genet Dev 2: 937-942.

Hyma, K.E., Lacher, D.W., Nelson, A.M., Bumbaugh, A.C., Janda, J.M., Strockbine, N.A., et al. (2005) Evolutionary genetics of a new pathogenic Escherichia species: Escherichia albertii and related Shigella boydii strains. J Bacteriol 187: 619-628.

Ingle, D.J., Clermont, O., Skurnik, D., Denamur, E., Walk, S.T., and Gordon, D.M. (2011) Biofilm formation by and thermal niche and virulence characteristics of Escherichia spp. Appl Environ Microbiol 77: 2695-2700.

Jaureguy, F., Landreau, L., Passet, V., Diancourt, L., Frapy, E., Guigon, G., et al. (2008) Phylogenetic and genomic diversity of human bacteremic Escherichia coli strains. BMC Genomics 9: 560.

Kaper, J.B., Nataro, J.P., and Mobley, H.L. (2004) Pathogenic Escherichia coli. Nat Rev Microbiol 2: 123-140.

Lawrence, J.G., Ochman, H., and Hartl, D.L. (1991) Molecular and evolutionary relationships among enteric bacteria. J Gen Microbiol 137: 1911-1921.

Le Gall, T., Clermont, O., Gouriou, S., Picard, B., Nassif, X., Denamur, E., and Tenaillon, O. (2007) Extraintestinal virulence is a coincidental by-product of commensalism in B2 phylogenetic group Escherichia coli strains. Mol Biol Evol 24: 2373-2384.

Lefort, A., Panhard, X., Clermont, O., Woerther, P.L., Branger, C., Mentre, F., et al. (2011) Host factors and portal of entry outweigh bacterial determinants to predict the severity of Escherichia coli bacteremia. J Clin Microbiol 49: 777-783. 
Lescat, M., Hoede, C., Clermont, O., Garry, L., Darlu, P., Tuffery, P., et al. (2009) aes, the gene encoding the esterase B in Escherichia coli, is a powerful phylogenetic marker of the species. BMC Microbiology 9: 723.

Luo, C., Walk, S.T., Gordon, D.M., Feldgarden, M., Tiedje, J.M., and Konstantinidis, K.T. (2011) Genome sequencing of environmental Escherichia coli expands understanding of the ecology and speciation of the model bacterial species. Proc Natl Acad Sci USA 108: 7200-7205.

Neidhardt, F.C., Curtiss, R., III, Ingraham, J.L., Lin, E.C.C., Low, K.B., Magasanik, B., et al. (1996) Escherichia Coli and Salmonella: Cellular and Molecular Biology. Washington, DC, USA: ASM Press.

Oaks, J.L., Besser, T.E., Walk, S.T., Gordon, D.M., Beckmen, K.B., Burek, K.A., et al. (2010) Escherichia albertii in wild and domestic birds. Emerg Infect Dis 16: 638-646.

Ochman, H., and Selander, R.K. (1984) Standard reference strains of Escherichia coli from natural populations. $J$ Bacteriol 157: 690-693.

Paradis, S., Boissinot, M., Paquette, N., Belanger, S.D., Martel, E.A., Boudreau, D.K., et al. (2005) Phylogeny of the Enterobacteriaceae based on genes encoding elongation factor Tu and F-ATPase beta-subunit. Int J Syst Evol Microbiol 55: 2013-2025.

Pham, H.N., Ohkusu, K., Mishima, N., Noda, M., Monir Shah, M., Sun, X., et al. (2007) Phylogeny and species identification of the family Enterobacteriaceae based on dnaJ sequences. Diagn Microbiol Infect Dis 58: 153-161.

Picard, B., Garcia, J.S., Gouriou, S., Duriez, P., Brahimi, N., Bingen, E., et al. (1999) The link between phylogeny and virulence in Escherichia coli extraintestinal infection. Infect Immun 67: 546-553.

Power, M.L., Littlefield-Wyer, J., Gordon, D.M., Veal, D.A., and Slade, M.B. (2005) Phenotypic and genotypic characterization of encapsulated Escherichia coli isolated from blooms in two Australian lakes. Environ Microbiol 7: 631-640.

Priest, F.G., and Barker, M. (2010) Gram-negative bacteria associated with brewery yeasts: reclassification of Obesumbacterium proteus biogroup 2 as Shimwellia pseudoproteus gen. nov., sp. nov., and transfer of Escherichia blattae to Shimwellia blattae comb. nov. Int J Syst Evol Microbiol 60: 828-833.

Rasko, D.A., Rosovitz, M.J., Myers, G.S., Mongodin, E.F., Fricke, W.F., Gajer, P., et al. (2008) The pangenome structure of Escherichia coli: comparative genomic analysis of E. coli commensal and pathogenic isolates. I Bacteriol 190: 6881-6893.

Sabarly, V., Bouvet, O., Glodt, J., Clermont, O., Skurnik, D., Diancourt, L., et al. (2011) The decoupling between genetic structure and metabolic phenotypes in Escherichia coli leads to continuous phenotypic diversity. J Evol Biol (in press): doi: 10.1111/j.1420-9101.2011.02287.x.

Savageau, M.A. (1983) Escherichia coli habitats, cell types, and molecular mechanisms of gene control. Am Nat 122: 732-744.

Skurnik, D., Ruimy, R., Andremont, A., Amorin, C., Rouquet, P., Picard, B., and Denamur, E. (2006) Effect of human vicinity on antimicrobial resistance and integrons in animal faecal Escherichia coli. J Antimicrob Chemother 57: 12151219.

Tenaillon, O., Skurnik, D., Picard, B., and Denamur, E. (2010) The population genetics of commensal Escherichia coli. Nat Rev Microbiol 8: 207-217.

Touchon, M., Hoede, C., Tenaillon, O., Barbe, V., Baeriswyl, S., Bidet, P., et al. (2009) Organised genome dynamics in the Escherichia coli species results in highly diverse adaptive paths. PLoS Genet 5: e1000344.

Walk, S.T., Alm, E.W., Gordon, D.M., Ram, J.L., Toranzos, G.A., Tiedje, J.M., and Whittam, T.S. (2009) Cryptic lineages of the genus Escherichia. Appl Environ Microbiol 75: 6534-6544.

Wirth, T., Falush, D., Lan, R., Colles, F., Mensa, P., Wieler, L.H., et al. (2006) Sex and virulence in Escherichia coli: an evolutionary perspective. Mol Microbiol 60: 1136-1151. 